\title{
Cardiología ajustada (Lean Cardiology). Eliminar los desperdicios para entregar una mejor calidad de atención
}

\author{
Dr. Gerardo Soca', Lic. Inés Prosper²
}

\author{
"No puede haber mejora del proceso \\ si no hay un proceso estandarizado." \\ Taiichi Ohno (1912-1990). Ingeniero industrial en Toyota.
}

\section{Introducción}

El management empresarial es algo intuitivamente muy alejado para los médicos. Para los profesionales de la salud, es más cómodo departir otro tipo de terminología (la estrictamente médica) sobre temas que son específicos "de la ciencia médica", o hasta leyendo y comentando casos clínicos. Esas acciones sin duda nos permiten llevar a cabo una mejor medicina, saciar el espíritu científico y contribuir a nuestro conocimiento personal (y también comunitario, si hablamos de los autores de artículos científicos).

La prestación de salud, como tantas actividades humanas, ha pasado por varias etapas en los últimos cien años. Varios paradigmas han cambiado en ese tiempo. Ha cambiado la definición de salud, el comportamiento del médico, la relación médico-paciente, el llamado "derecho médico", el tratamiento de los datos sensibles del paciente y, también, la relación del personal de salud con la administración de los recursos de los que dispone para realizar su tarea.

Así, toma impulso un estudio más formal sobre la manera de gestionar, dirigir y gobernar las empresas "proveedoras de salud", al tiempo que la salud pretende tener una cobertura universal, aumentan los costos por paciente tratado, y también aumenta la competencia entre instituciones médicas. El objetivo de este estudio es el mismo que cuando leemos una revista de medicina: servir mejor.

Las condiciones actuales del sector salud (enu- meradas anteriormente) fuerzan a que los administradores de recursos (personal no médico, médicos, directivos médicos) se centren en mejorar los resultados clínicos, "la experiencia" del paciente y su seguridad, a la vez que se contienen los costos económicos. Lo mismo puede ser visto desde la óptica del paciente, quien cada vez es más demandante de una buena calidad de atención, dentro de las opciones de su posible cobertura.

Desde los años 90, en el sector de la salud ha tomado impulso la aplicación de estrategias de administración de recursos derivadas de otras áreas. En particular, el método de producción de Toyota (TPS: Toyota Production System) ha sido tomado por infinidad de disciplinas. Este método fue moldeado por autores estadounidenses ${ }^{(1,2)} \mathrm{y}$ rebautizado como Lean ('esbelto', 'magro' o, mejor aún, 'ajustado') y aplicado a diversas áreas, como las empresas de salud (Lean Healthcare). La idea de esta corriente es muy sencilla, así como sus herramientas de implementación.

Aumentar el valor para el cliente, eliminando los desperdicios del proceso asistencial

Valor se define como cualquier actividad que contribuya de manera directa a satisfacer las necesidades del cliente. Desperdicio se define como cualquier actividad distinta de los recursos absolutamente esenciales para añadir valor al cliente.

En Lean Healthcare, el cliente final es el paciente, pero para implementar este sistema a todo nivel hay que tener en cuenta el cliente interno de cada proceso.

Podemos pensar que lo anterior es obvio, y que no hay actividades estériles en nuestra práctica. ¿Cómo es que una empresa de salud pública o pri- 
vada, con una estructura compleja, podría, de forma sistemática, realizar actividades que no aportan absolutamente nada a su cliente, o que pongan en peligro a los pacientes, o hagan de su asistencia una experiencia desagradable, provocando cansancio y frustración entre el personal de salud?

Expertos en administración de procesos estiman que $60 \%$ de toda la operativa de un centro asistencial no aporta ningún valor, e incluso que una parte sustancial tiene efecto negativo. Una de las razones es que el médico es formado en conocimientos y destrezas, y luego ejerce su profesión con total autonomía. Tras su formación, de él solo se espera "que actúe de la mejor forma", aun cuando se encuentre inmerso en un sistema a veces poco diseñado, o con un diseño que permanece incambiado desde hace décadas.

\section{Un ejemplo asistencial}

Supongamos que una paciente debe concurrir a policlínica cardiológica por una patología que le provoca síntomas severos relacionados con la presencia de insuficiencia cardíaca. Espera turno por dos semanas. El día de la consulta, espera (en la "sala de espera") durante 90 minutos para acceder a su atención con el cardiólogo. En 10 minutos, el especialista le explica su diagnóstico e indica estudios. Se le da fecha para dos días distintos para realizarse la paraclínica. Con los resultados de dichos estudios, es vista luego por cirujano cardíaco tras otra espera, ya que todos los pacientes fueron agendados a la misma hora. El cirujano le solicita más estudios, y luego de algunas semanas, se le realiza un cateterismo cardíaco preoperatorio, cuyo resultado es normal. Se traslada a domicilio, porque la cirugía aún no está autorizada, y es coordinada unos días después. Tiene buena evolución en la UCI, pero su traslado a piso se retrasa dos días por falta de cama. Prosigue bien, y es dada de alta a domicilio. Tiene otras consultas de control posoperatorio, todas con las mismas molestias (esperas prolongadas, movimientos innecesarios, estudios que se realizan en días diferentes). A pesar de que su patología ha sido resuelta, queda asintomática y prolonga considerablemente su sobrevida, la paciente evalúa toda su experiencia asistencial como "mala".

Este ejemplo ficticio no está muy lejos de la realidad. El proceso descrito está lleno de desperdicios (retrasos, sobreproducción, movimientos, traslados, esperas) y sujeto a una enorme variabilidad, así como cualquier otro de nuestra práctica médica habitual.

- ¿Cuáles de todas estas actividades eran de

\begin{tabular}{|c|c|c|}
\hline & Desperdicio & Ejemplo \\
\hline 1. & $\begin{array}{l}\text { Exceso de } \\
\text { producción. }\end{array}$ & $\begin{array}{l}\text { Exámenes } \\
\text { diagnósticos } \\
\text { innecesarios. }\end{array}$ \\
\hline 2. & Transporte. & $\begin{array}{l}\text { De pacientes o } \\
\text { productos. }\end{array}$ \\
\hline 3. & Inventario. & $\begin{array}{l}\text { Stocks de materiales } \\
\text { médicos. }\end{array}$ \\
\hline 4. & Tiempo de espera. & $\begin{array}{l}\text { Colas de espera, listas } \\
\text { de espera. }\end{array}$ \\
\hline 5. & Reprocesamiento. & $\begin{array}{l}\text { Reintervención } \\
\text { quirúrgica de paciente }\end{array}$ \\
\hline 6. & Defectos. & $\begin{array}{l}\text { Quejas, tratamiento } \\
\text { incompleto. }\end{array}$ \\
\hline 7. & Movimientos. & $\begin{array}{l}\text { Es personal } \\
\text { moviéndose en busca } \\
\text { de materiales o de } \\
\text { documentos. }\end{array}$ \\
\hline 8. & $\begin{array}{l}\text { Desperdicio del } \\
\text { talento. }\end{array}$ & $\begin{array}{l}\text { Profesionales médicos } \\
\text { desaprovechados. }\end{array}$ \\
\hline
\end{tabular}

innegable valor para la paciente? Podríamos resumirlas como las que permiten "llegar a un diagnóstico y operarse", para solucionar, de esa forma, sus síntomas.

- ¿Qué actividades no son de valor para ella, pero resultan necesarias? Realizarse el cateterismo cardíaco y la evaluación paraclínica, acceder a la internación. Estas actividades deben ser minimizadas en extensión y molestias para el paciente.

- ¿Cuáles son las actividades sin valor y, además, innecesarias? Las esperas, las molestias y los traslados innecesarios, así como el reprocesamiento. Estas actividades deben ser eliminadas.

Es posible ofrecer mejores servicios, en forma más rápida, efectiva y a menor costo mediante la eliminación de los desperdicios y la variabilidad (falta de estandarización), lo que puede lograrse por diseño, usando diversas herramientas.

\section{Identificación de los desperdicios}

Es un concepto fundamental del Lean Management y del TPS, ya que su eliminación es el medio para conseguir mejores resultados, para así usar todos los recursos en generar más actividades de valor. Se describen ocho tipos de desperdicios en la elaboración de productos o la prestación de servicios (tabla 1 ).

Flujo de valor

Una vez identificado qué es lo que implica el va- 


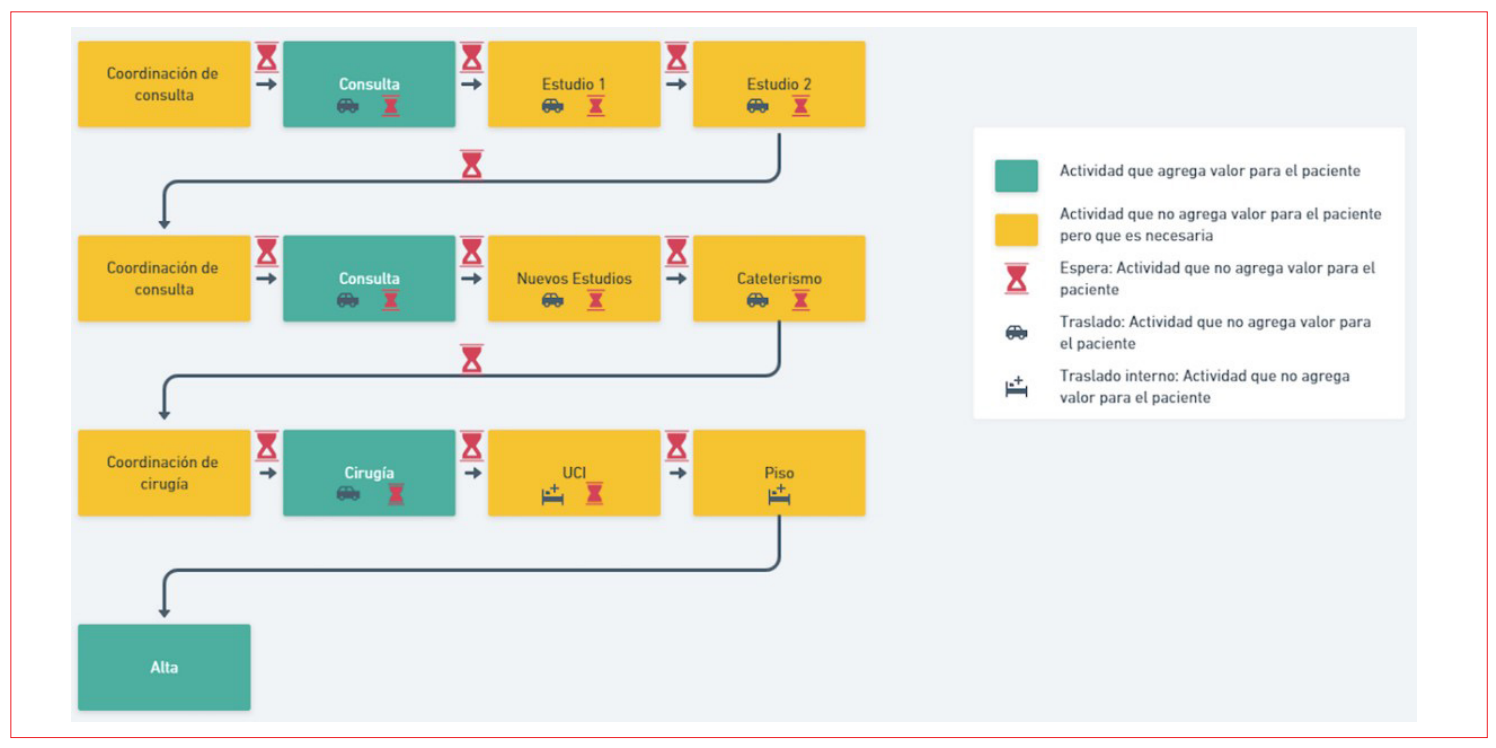

Figura 1. Flujograma según valor de las actividades del proceso descrito.

lor para el paciente, podemos clasificar las actividades del proceso asistencial o terapéutico según las actividades de valor, las necesarias y las innecesarias, e identificar en el diagrama de flujo del proceso estas actividades y la proporción del tiempo de proceso insumido (figura 1).

Dependiendo de la actividad analizada, y del nivel organizacional en el que pueda ser resuelta, serán las medidas a tomar para priorizar las actividades de valor, minimizar la molestia de las actividades necesarias, y erradicar las actividades innecesarias.

Un miembro del directorio de una mutualista no podrá realizar todas las acciones que eliminen estas actividades sin valor (solo las referentes al gobierno de la institución). Un funcionario de la primera línea de atención tampoco podrá tomar todas las decisiones (solo las referentes a la ejecución de las actividades, al aporte de nuevas ideas de operación y a la gestión cotidiana de los recursos). Se desprende así que, para llevar a cabo este "esencialismo" de provisión de salud, todos los niveles de la organización deben estar comprometidos, o al menos alineados con coherencia tras un ideal que consiste en usar todos los recursos disponibles de la institución en aumentar las actividades de valor para los pacientes.

\section{Jidoka}

Con este término japonés se designa a la evidenciación total, y durante el propio proceso, de los desperdicios y defectos de calidad. Como ejemplo, en la producción de automóviles en una planta de Toyota, el control de calidad es realizado por todos los operarios durante todo el proceso, y si existe el más mínimo desperfecto, la producción de la planta se detiene en forma completa hasta la resolución definitiva del problema, lo que puede implicar una reunión de varias personas, incluyendo operarios, supervisores, jefes y directores. Esta detención total de la línea de producción se produce de decenas a cientos de veces por día. El resultado es la mayor calidad posible, y un proceso de mejora en evolución permanente.

En un proceso médico asistencial, cada uno de los profesionales de la salud implicados es un "cliente" del paso anterior del proceso (cliente interno). La filosofía Lean establece que a cada cliente interno debe entregársele un producto (paciente) con la máxima calidad definida por el proceso. De esta forma, el control de calidad se da durante toda la asistencia. Por consecuencia, no se continúa el proceso si no se entrega la calidad predefinida: "Si no está perfecto, no lo pases al siguiente paso, si no está perfecto, no lo recibas del paso anterior".

Para evidenciar los defectos de calidad, cada tarea debe estar específicamente definida (estandarizada): qué pasos deben seguirse, cuándo y quién debe hacerlo, qué resultado se espera.

Mediante jidoka se evidencian con facilidad las fallas de la asistencia cuando se produce un alejamiento de la definición estandarizada. En ese momento, la "producción" (asistencia) se detiene para solucionar esa situación, y se busca la causa raíz del problema para que no sea posible que ocurra de nuevo. Este concepto es de difícil implementación en la salud. Todos los actuantes 


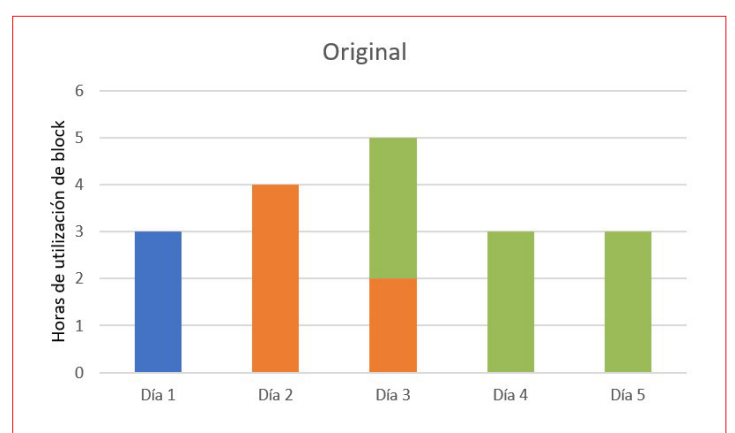

Figura 2. Actividad sin diseño.

en la asistencia de un paciente "tratan de hacer lo mejor", tradicionalmente el reporte de errores propios o de terceros está penado o mal visto, o no activa acciones correctivas a futuro (a diferencia de otras áreas, como la industria aeronáutica), y entran en juego diversos factores como el ego profesional y la competencia entre pares, entre otros. Todo esto atenta contra la mejora continua del proceso.

En la práctica médica, jidoka puede verse desde el apósito transparente que permite evaluar el estado de una herida, el ecocardiograma intraoperatorio que demuestra que una prótesis valvular funciona perfectamente antes de pasar el paciente al próximo "cliente interno", o hasta el compromiso total de una institución médica con el reporte voluntario y no punitivo de errores o eventos adversos, entre otras medidas de mejora. Igualmente, estas son medidas puntuales, y un centro de salud Lean debe evidenciar sus oportunidades de mejora en forma constante.

\section{Heijunka}

Este término se refiere a la nivelación de la producción de un bien físico o de la prestación de un servicio. En medicina podríamos poner como ejemplo un centro quirúrgico que opera en tres niveles de complejidad de procedimientos, con diferente consumo de horas de quirófano, y en un block en el que hay que pagar horas extra, por ejemplo, luego de la cuarta hora de duración de una cirugía (a un alto precio y restando recursos humanos a otras áreas). Sin diseño, los pacientes podrían operarse de una forma eficaz, pero que determine ineficiencias y restricciones (cuellos de botella) en los siguientes pasos del proceso (por ejemplo, falta de camas de UCI o falta de personal para cubrir horas extra, que además generan costos extra) (figura 2). Mediante nivelación diseñada (figura 3), podrían intervenirse de manera alternativa los pacientes de diferentes niveles de complejidad, para no saturar ningún paso posterior y disminuirse los desperdicios. Podemos apli-

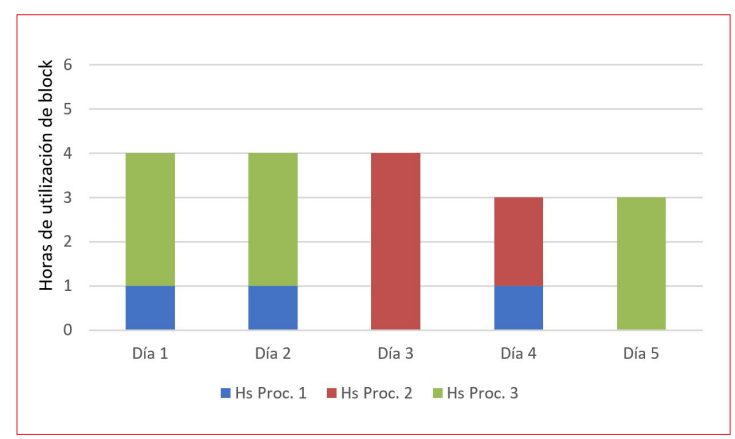

Figura 3. Actividad con nivelación diseñada (heijunka).

car este concepto en cualquier proceso de coordinación de procedimientos, consultas médicas y estudios.

Otro ejemplo sencillo y cotidiano es la forma en la que se citan los pacientes a una consulta. Si se citan todos los pacientes al principio de la consulta (figura 4), los primeros en ser atendidos no tendrán casi espera, en cambio, los últimos tendrán una larga espera (desperdicio), lo que generará molestias. A su vez, hay que disponer de espacio y sillas para todos los pacientes citados, y el personal de recepción se verá saturado al inicio de la consulta y luego estará ocioso. Por el contrario, se puede aplicar heijunka citando a los pacientes a intervalos regulares (figura 5). De esta forma, la espera de todos los pacientes será similar, la ocupación del personal de recepción será similar a lo largo de toda la consulta, y el espacio de espera del que tendremos que disponer será significativamente menor.

\section{Restricción sistemática de errores que causan desperdicios}

Los errores en medicina son frecuentes, esperables y, en su mayoría, previsibles. Existen varias herramientas que pueden utilizarse para la gestión de errores, cuyo primer objetivo es la eliminación de la posibilidad de que exista un error. Se denomina poka yoke (literalmente 'a prueba de errores') al diseño físico de los elementos (instalaciones, instrumental, etc.) de forma que el error no sea posible (por ejemplo, imposibilidad de transitar un molinete en el sentido contrario, conectar un ventilador a la aspiración central o el aspirador de la sala de operaciones a la salida del oxígeno). Ante un error posible, a veces sistemático, podemos hacer una modificación física tendiente a erradicarlo.

\section{Mejores prácticas}

El auge de la Medicina Basada en la Evidencia ha permitido mejorar los resultados clínicos. En la actualidad, es difícil mantenerse totalmente actualizado, por la frecuencia de publicación de 


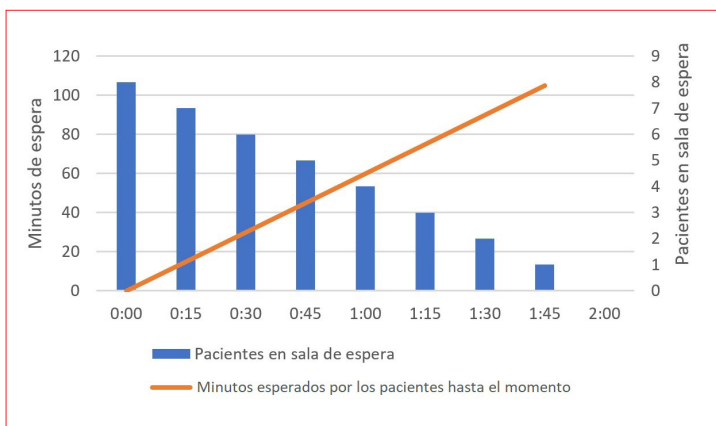

Figura 4. Todos los pacientes son citados al inicio de la consulta.

ensayos clínicos randomizados. Esto es atenuado por la publicación de guías de práctica clínica. Sin embargo, existe una brecha cada vez más grande entre las guías y su aplicación en la práctica clínica (brecha terapéutica), y se postula que, cuanto mayor es la brecha de un centro o país, peores son sus resultados clínicos (12\% a $79 \%$ de los actos médicos en EE. UU. están por fuera de las guías de práctica clínica) ${ }^{(3)}$. La falta de aplicación de las mejores prácticas terapéuticas es considerada la quinta causa de muerte en EE. UU. Las metodologías Lean y otras del estilo proveen un entorno para disminuir la brecha terapéutica, ya que en general aplican estos conceptos: a) abordaje sistemático y estandarizado de las actividades (en contraposición al antiguo paradigma del médico, que a través de su propio criterio resolverá cada paciente), b) medir continuamente variables de interés que permitan detectar un problema, testear cambios y monitorear las acciones, y c) un fuerte componente humano centrado en el paciente. Así, otra herramienta para hacer una mejor medicina es la adhesión sistemática, diseñada y obligatoria a las mejores prácticas demostradas en el procedimiento médico en cuestión.

\section{Reflexión final}

Puede existir escepticismo sobre la aplicación de un método de la industria automotriz a la práctica médica. Lean es aplicado a turismo, tecnología, manufactura y, obviamente, a salud, como el icónico caso del Virginia Mason Medical Center (Seattle, EE. UU.) que implantó este programa en 2002 y es modelo a nivel mundial ${ }^{(4)}$, así como otros denotados casos de éxito ${ }^{(5,6)}$.

Todos ganan si logramos trabajar en eficiencia y calidad de la asistencia médica (pacientes, sistema de salud y profesionales). Lean es un proceso dinámico y enfocado en el paciente, por el cual las personas que trabajan en una empresa médica continuamente eliminan los desperdicios y crean

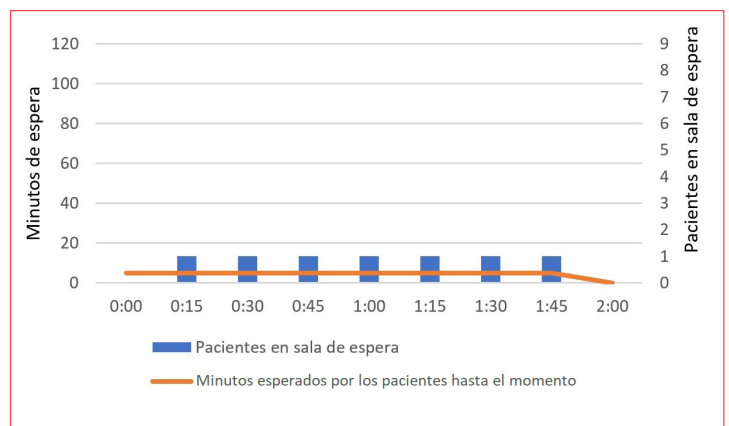

Figura 5. Los pacientes son citados cada 15 minutos. Se trata de una consulta de 2 horas con un tiempo de atención promedio de 15 minutos por paciente.

valor. Es un camino, no un lugar al que llegar. Los objetivos pueden ser en apariencia contradictorios (menos personal abocado a una tarea, menor tiempo de espera de pacientes y disminución de costos y mejora de calidad). Sin embargo, la eliminación de los desperdicios lo hace posible. Un liderazgo alineado es esencial, puesto que con los dogmas directivos tradicionales es difícil sostener una estrategia Lean. La dirección debe empoderar a los profesionales médicos y no médicos de primera línea, para que desarrollen una faceta de mejora continua para resolver problemas, involucrar a cada persona del equipo asistencial y respetar y tomar en cuenta muchas opiniones, experiencias y habilidades.

Esta filosofía de trabajo y sus herramientas son aplicables a grandes empresas de salud, a pequeñas unidades asistenciales e incluso a la práctica clínica individual, si la actividad lo permite. Solo se trata de eliminar lo innecesario para centrarse en lo valioso para el paciente. ¿Qué podría salir mal?

\section{Gerardo Soca, ORCID 0000-0002-8073-3292 \\ Inés Prosper, ORCID 0000-0001-9120-0101}

Este artículo fue aceptado para su publicación por el Editor jefe, Dr. Federico Ferrando

\section{Bibliografía}

1. Wikipedia Contributors. John Krafcik. Wikipedia, The Free Encyclopedia. October 13, 2021. Disponible en: https://en.wikipedia.org/w/index.php?title=John_Krafcik\&oldid=1049787071. [Consulta: 8 Set 2021].

2. Womack J, Jones D. Lean thinking: Banish waste and create wealth in your corporation. J Oper Res Soc. 1996; 48(11): 1144-50. Doi: 10.1057/palgrave. jors. 2600967

3. Mentz R, Hernandez A, Berdan L, Rorick T, O'Brien E, Ibarra J, et al. Good clinical practice guidance and pragmatic clinical trials: balancing the best of both worlds. Circulation. 2016; 133(9):872-80. doi: 10.1161/CIRCULATIONAHA.115.019902 
4. Virginia Mason Institute. What is lean healthcare? Seattle: Virginia Mason Institute, 2021. Disponible en: https://www.virginiamasoninstitute.org/what-is-lean-health-care/. [Consulta: 8 Set 2021].

5. Piggott Z, Weldon E, Strome T, Chochinov A. Application of lean principles to improve early cardiac care in the emergency department. CJEM. 2011; 13(5):325-32. doi: 10.2310/8000.2011.110284

6. Breen L, Trepp RJr, Gavin N. Lean process improvement in the Emergency Department. Emerg Med Clin North Am. 2020; 38(3):633-46. doi: 10.1016/j.emc.2020.05.001 